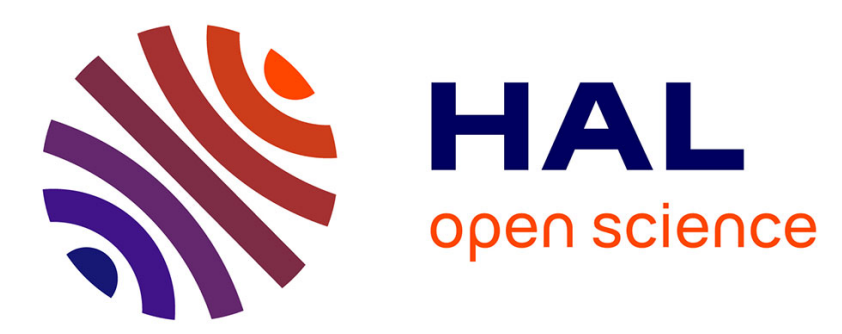

\title{
Bare-Metal Reservation for Cloud: an Analysis of the Trade Off between Reactivity and Energy Efficiency
}

Marcos Dias de Assuncao, Laurent Lefèvre

\section{To cite this version:}

Marcos Dias de Assuncao, Laurent Lefèvre. Bare-Metal Reservation for Cloud: an Analysis of the Trade Off between Reactivity and Energy Efficiency. Cluster Computing, 2018, 21, pp.1289-130. 10.1007/s10586-017-1094-y . hal-01571288

\section{HAL Id: hal-01571288 \\ https://hal.inria.fr/hal-01571288}

Submitted on 2 Aug 2017

HAL is a multi-disciplinary open access archive for the deposit and dissemination of scientific research documents, whether they are published or not. The documents may come from teaching and research institutions in France or abroad, or from public or private research centers.
L'archive ouverte pluridisciplinaire HAL, est destinée au dépôt et à la diffusion de documents scientifiques de niveau recherche, publiés ou non, émanant des établissements d'enseignement et de recherche français ou étrangers, des laboratoires publics ou privés. 


\title{
Bare-Metal Reservation for Cloud: an Analysis of the Trade Off between Reactivity and Energy Efficiency
}

\author{
Marcos Dias de Assunção • Laurent Lefèvre
}

Received: - / Accepted: -

\begin{abstract}
In this work, we investigate factors that can impact the elasticity of bare-metal resources. We analyse data from a real bare-metal deployment system to build a deployment time model, then use it to determine how long it takes to deliver requested resources to cloud users. Simulation results show that reservations can help reduce the time to deliver a provisioned cluster to its customer, by enabling machines to be started in advance or be kept powered on when there are impending reservations. Such an approach, when compared to strategies that switch-off idle resources, shows that similar energy savings can be achieved with much smaller impact on the time to deliver the provisioned clusters.
\end{abstract}

\section{Introduction}

Cloud computing has become a popular model for providing IT resources and services to organisations of all sizes [1]. The workload consolidation that clouds provide by virtualising resources and enabling customers to share the underlying physical infrastructure brings benefits such as energy efficiency and better system utilisation. Most cloud providers enable their customers to request resources on demand and pay for their use on a per-hour basis. Such elasticity allows for adjusting the allocated capacity dynamically to meet fluctuating demands.

Though this consolidated model suits most of the today's use cases, certain applications such as those that demand High Performance Computing (HPC) or specialised resources, are not fully portable to this scenario

Inria Avalon, LIP Laboratory

École Normale Supérieure de Lyon

University of Lyon, France

E-mail: \{name.surname\}@ens-lyon.fr as they are generally resource intensive and sensitive to performance variations. Many applications still demand homogeneity among computing nodes and predictable network performance. Moreover, certain customers prefer bare-metal resources over shared ones for security reasons. The means used by cloud providers to offer customers with high and predictable performance mostly consist in deploying bare-metal resources or grouping Virtual Machines (VMs) where high network throughput and low latency can be guaranteed. This model contrasts with traditional cloud use cases as it is costly and provides little flexibility regarding workload consolidation and resource elasticity. Using public clouds or co-locating HPC applications on the same physical hardware, however, have proven difficult [16, 21,30, 33].

Over the past, HPC users have been tolerant to the time needed for resources to become available as they generally share large clusters to which exclusive access is made by submitting a job that may wait in queue for a period often longer than the job execution itself. Users of bare-metal services also commonly accept provisioning delays that can vary from hours to several days. We do not consider that clouds should adopt a similar queuing model, but we believe that a compromise between wait time and on-demand access could be exploited for bare-metal resources in the cloud via resource reservations. Reservations provide means for reliable allocation and allow customers to plan the execution of their applications, which is key to many use cases that require bare-metal and specialised resources. Current reservation models of public clouds rely on requesting resources in advance for a long period (i.e. from one to three years) or bidding for virtual machine instances in a spot market.

In this work, we analyse historical data on the provision of bare-metal resources from a real system and 
attempt to model the time required by bare-metal deployment. Results from discrete-event simulations then demonstrate (i) the energy-saving potential of strategies that switch off unused resources and (ii) how reservations can help reduce the time to deliver provisioned clusters to their customers, by enabling servers to be started in advance or be kept powered on when there are impending reservations. In summary, the main contributions of this paper are to:

- Present an analysis on deployment of bare-metal resources and model the deployment time.

- Evaluate the impact of reservations on the time to deliver clusters of bare-metal resources to customers and the potential energy savings.

The rest of this paper is organised as follows. Section 2 presents background on bare-metal provisioning and the motivation for resource reservation of bare-metal resources in the cloud. The analysis of historical data on bare-metal deployment and the model are presented in Section 3. Section 4 describes reservation strategies, whereas Section 5 presents the experimental setup and obtained results. Section 6 discusses related work on resource reservations, whereas Section 7 concludes the paper.

\section{Background and Motivation}

Grid'5000 [4] — an experimental platform comprising several sites in France and Luxembourg — provides users with single-tenant environments enabled by baremetal provisioning and reservations. To utilise the platform, a user can either make a request for an advance reservation of computing resources or submit a besteffort request which grants the user access to required resources whenever they become available. Reservations take precedence over best-effort requests so that a besteffort job can be cancelled if its resources are reserved by another user.

We considered a simple experiment to estimate the impact of reservations on the utilisation of a data centre. We used the advance-reservation log from the scheduler [5] of a Grid'5000 site (i.e. the data centre located in Lyon) and simulated a scheduling under two scenarios: the current scheduling where users make reservations to use resources at a time in the future; and an extrapolation where all requests are treated as immediate reservations where resources are allocated as they arrive, hence emulating a more elastic cloud-like scenario. Figure 1 shows the number of cores required to handle requests over time under each scenario. Although this is an extreme scenario where all requests are treated as they arrive, we believe that if users knew resources were available whenever they wanted, certain users who currently reserve resources in advance would have changed their behaviour; particularly users that currently use the platform over weekends or at night.

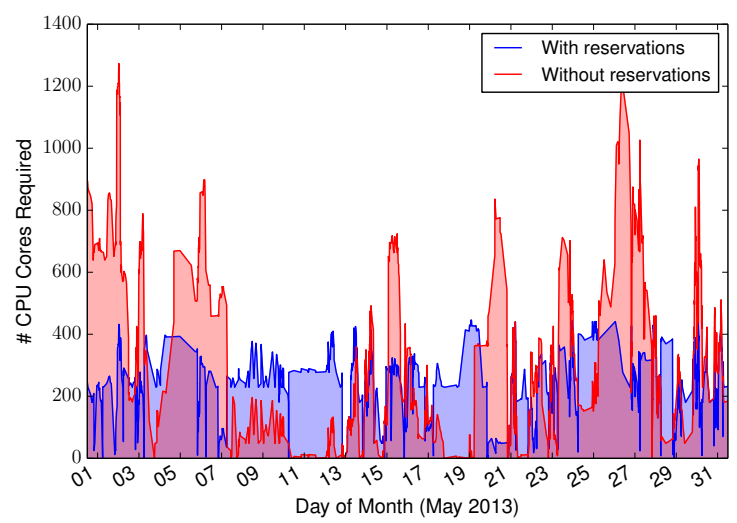

Fig. 1 Maximum number of CPUs needed at the Grid'5000 Lyon site.

Grid'5000 is an experimental platform, but studies of other system logs revealed similar bursts of requests during working hours [9]. Providing bare-metal or specialised VMs with the elasticity with which cloud customers are familiar can be costly, and reservations may be explored to help minimise these costs.

\section{Modelling Bare-Metal Deployment}

Workload traces that provide information on the time taken to perform operations required to deploy resources, such as switching resources on, cloning operating system images, and partitioning physical disks are difficult to come by. To model the time required for deployment, we use traces gathered from Grid'5000 which have been generated by Kadeploy3 [14]; a disk imaging and cloning tool that takes a file containing the operating system to deploy (i.e. an environment) and copies it to target nodes. An environment deployment by Kadeploy3 consists of three phases:

1. Minimal environment setup, where nodes reboot into a minimal environment with tools for partitioning and disks and creating the necessary file systems.

2. Environment installation, when the environment is broadcast and copied to all nodes, and post-copy operations are performed.

3. Reboot of nodes using the deployed environment. 
We gathered several years of Kadeploy3 traces from five clusters on three Grid'5000 sites and evaluated the time to execute the three phases described above. Table 1 details the considered clusters.

Table 1 Clusters whose deployment logs were considered.

\begin{tabular}{|c|c|c|c|}
\hline $\begin{array}{l}\text { Cluster } \\
\text { Name }\end{array}$ & $\begin{array}{c}\# \\
\text { Nodes }\end{array}$ & $\begin{array}{c}\text { Install } \\
\text { Date }\end{array}$ & $\begin{array}{c}\text { Node } \\
\text { Characteristics }\end{array}$ \\
\hline parapluie & 40 & Oct. 2010 & $\begin{array}{l}2 \text { CPUs AMD } 1.7 \mathrm{GHz}, \\
12 \text { cores/CPU, } 48 \mathrm{~GB} \\
\text { RAM, 232GB DISK }\end{array}$ \\
\hline parapide & 25 & Nov. 2011 & $\begin{array}{l}2 \text { CPUs Intel } 2.93 \mathrm{GHz} \text {, } \\
4 \text { cores/CPU, } 24 \mathrm{~GB} \\
\text { RAM, } 465 \mathrm{~GB} \text { DISK }\end{array}$ \\
\hline paradent & 64 & Feb. 2009 & $\begin{array}{l}2 \text { CPUs Intel } 2.5 \mathrm{GHz} \\
4 \text { cores/CPU, 32GB } \\
\text { RAM, 298GB DISK }\end{array}$ \\
\hline stremi & 44 & Jan. 2011 & $\begin{array}{l}2 \text { CPUs AMD } 1.7 \mathrm{GHz}, \\
12 \text { cores/CPU, } 48 \mathrm{~GB} \\
\text { RAM, 232GB DISK }\end{array}$ \\
\hline sagittaire & 79 & Jul. 2007 & $\begin{array}{l}2 \text { CPUs AMD } 2.4 \mathrm{GHz} \text {, } \\
1 \text { core/CPU, } 1 \mathrm{~GB} \\
\text { RAM, 68GB DISK }\end{array}$ \\
\hline
\end{tabular}

We considered all deployments from Jan. 2010 through Dec. 2013. The first step towards building a model consisted in creating time histograms and visually examining probability distributions that were likely to fit the data. Scott's method was used to determine the size of histogram bins [24]. After considering some distributions, we found that log-normal, gamma and generalised gamma were most likely to fit the data. Figure 2 depicts the results of fitting these distributions to the deployment time information of each cluster. In general, deployment presents an average completion time with occasional failures overcome by executing other routines and performing additional server reboots.

The goodness of fit of the distributions has also been submitted to the Kolmogorov-Smirnov test (KS test), whose D-statistic quantifies the distance between the distribution function of empirical values and the cumulative distribution function of the reference distribution. Although the results summarised in Table 2 do not differ much, log-normal provides slightly better fit to most clusters, and is hence used to model deployment time.

We also have analysed how the deployment time changes according to the number of machines simultaneously configured per deployment (i.e. the deployment size). Figure 3 summarises the information on deployment for several deployment sizes and the considered clusters. The bubbles illustrate the distribution of deployments across the intervals of required numbers of
Table 2 Kolmogorov-Smirnov test for goodness of fit.

\begin{tabular}{cccc}
\hline \multirow{2}{*}{$\begin{array}{c}\text { Cluster } \\
\text { Name }\end{array}$} & \multicolumn{3}{c}{ D-Statistics } \\
\cline { 2 - 4 } & Log-normal & Gamma & Gen. Gamma \\
\hline parapluie & 0.051 & 0.066 & 0.059 \\
\hline parapide & 0.111 & 0.095 & 0.091 \\
\hline paradent & 0.041 & 0.046 & 0.043 \\
\hline stremi & 0.051 & 0.036 & 0.039 \\
\hline sagittaire & 0.067 & 0.076 & 0.070 \\
\hline
\end{tabular}

machines, whereas Table 3 presents the number of deployments in the largest and smallest groups for each cluster.

Table 3 Number of deployments per group.

\begin{tabular}{ccc}
\hline \multirow{2}{*}{$\begin{array}{c}\text { Cluster } \\
\text { Name }\end{array}$} & \multicolumn{2}{c}{ Number of Deployments } \\
\cline { 2 - 3 } & Largest Group & Smallest Group \\
\hline parapluie & 9722 & 143 \\
\hline parapide & 6654 & 235 \\
\hline paradent & 10000 & 9 \\
\hline stremi & 6939 & 21 \\
\hline sagittaire & 9271 & 5 \\
\hline
\end{tabular}

The violin graphs in Figure 3 show that, with a few exceptions, the time taken by most deployments lies between the mean and the lower outliers, hence demonstrating the tendency that deployment time exhibits a lognormal behaviour. Moreover, the average deployment time increases proportionally to deployment size, which is expected as multiple simultaneous data transfers are carried out (e.g. copy of the OS image to nodes), hence competing for network resources. The bubbles show that most deployments require between 1 and 4 machines, while the corresponding violin graphs indicate that these deployments present the largest variations in deployment time. Further investigation revealed that this behaviour stems from the fact that Grid'5000 is an experimental platform. Most users, when creating customised environments and testing their deployments, do so by experimenting with a small number of machines (from 1 to 5). Under certain cases, some deployment phases are retried a few times before the request is considered successful.

Figure 4 shows the daily mean deployment time and the number of deployments for the considered clusters. One can notice a significant number of deployments for a few days. Further investigation revealed that these cases result from automated tests performed after updates of the bare-metal deployment system and plat- 

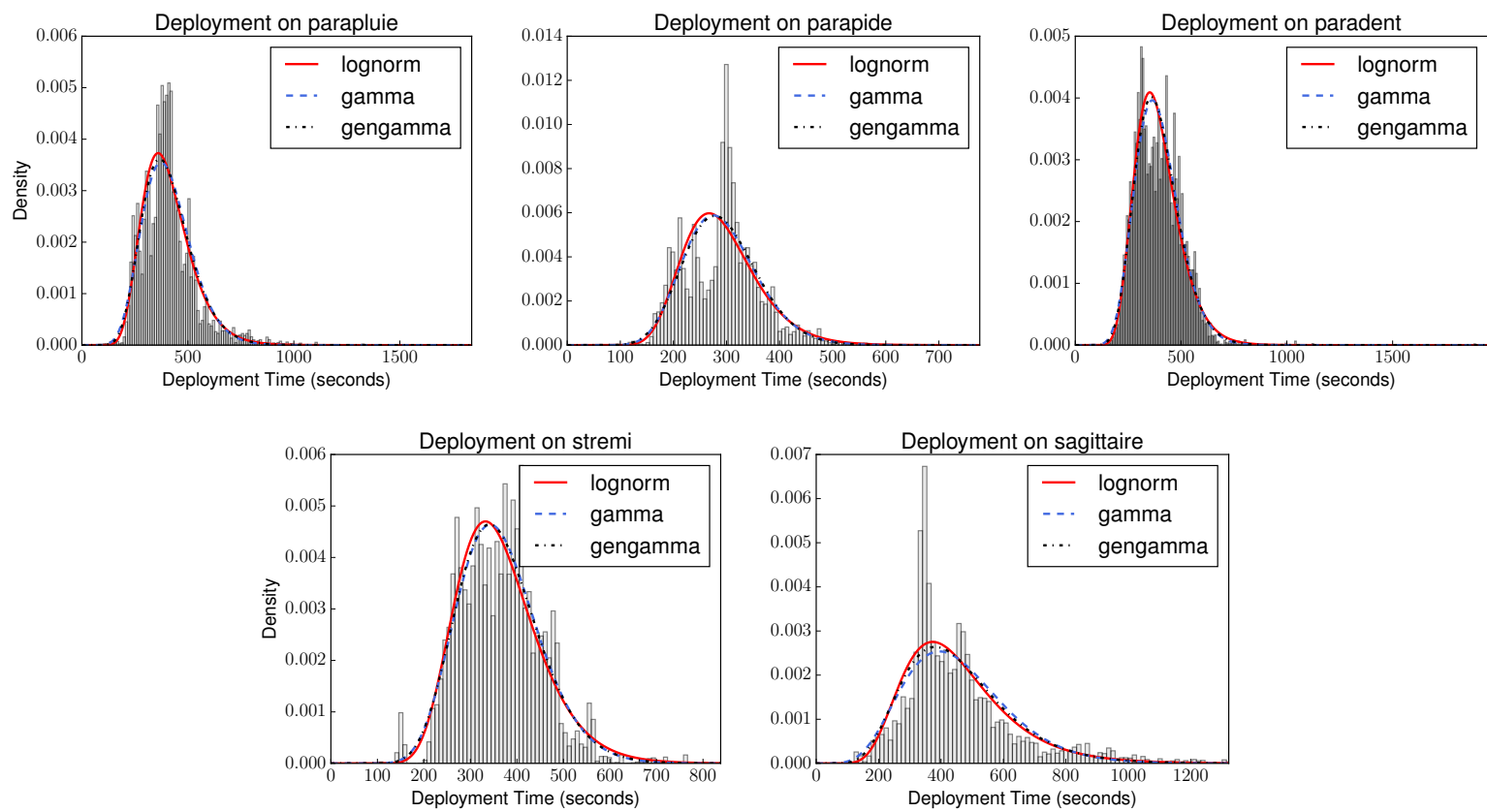

Fig. 2 Deployment time histograms and distribution fitting.
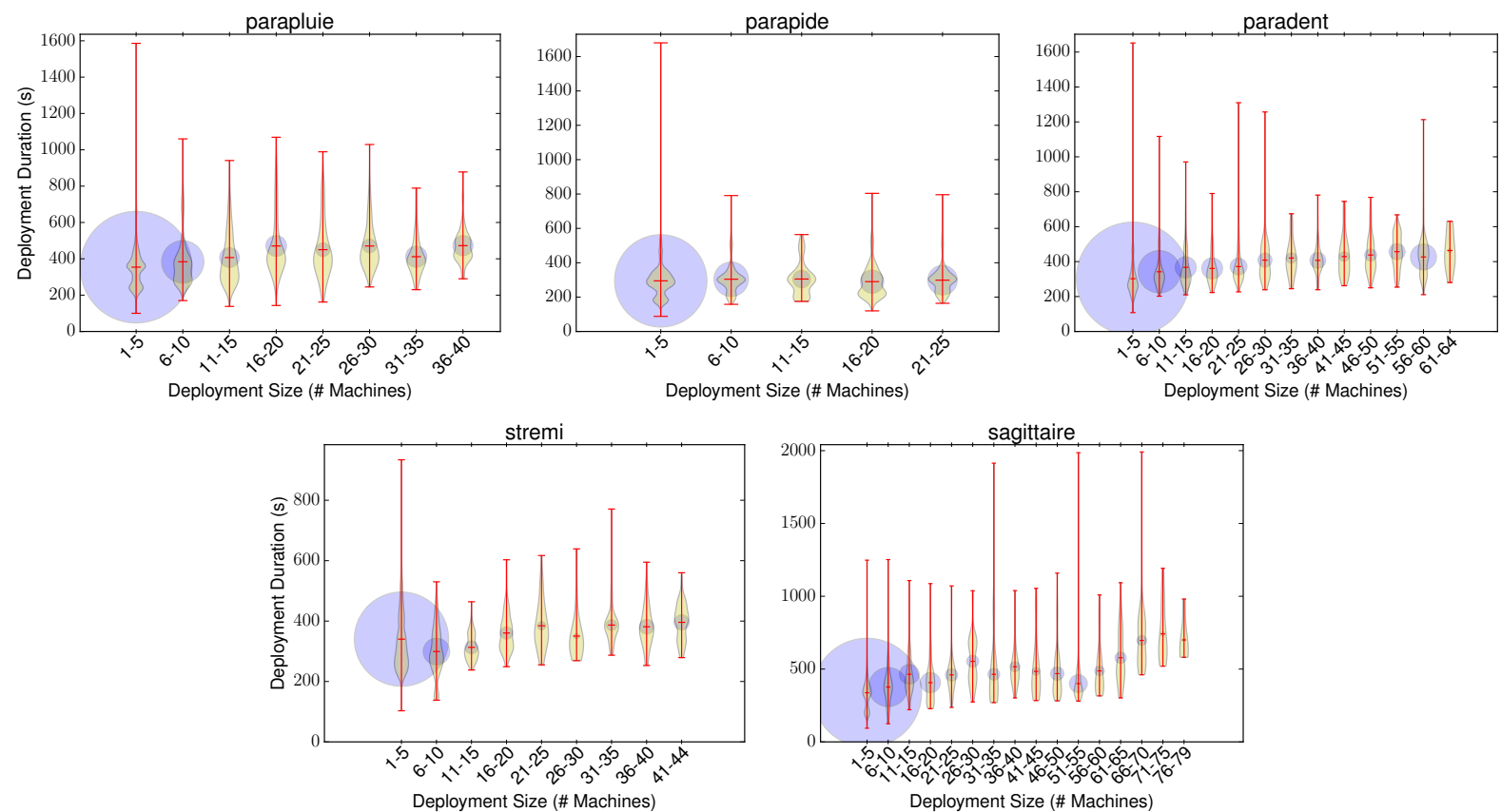

Fig. 3 Deployment time under various numbers of simultaneously configured machines; the bubbles illustrate the number of deployments in each interval.

form maintenance. Moreover, for certain clusters (e.g. parapluie, parapide and sagittaire) the daily mean deployment time decreases substantially from July 2012. After discussions with engineers responsible for the platform maintenance, we discovered that over time there had been changes in the last phase of environment deployment performed by Kadeploy. Instead of perform- ing a full system reboot to initialise the newly deployed environment, some machines utilise $k e x e c^{1}$ to load the new Linux kernel into memory and boot the new system, hence avoiding a hardware reboot and its firmware stage.

\footnotetext{
1 https://en.wikipedia.org/wiki/Kexec
} 

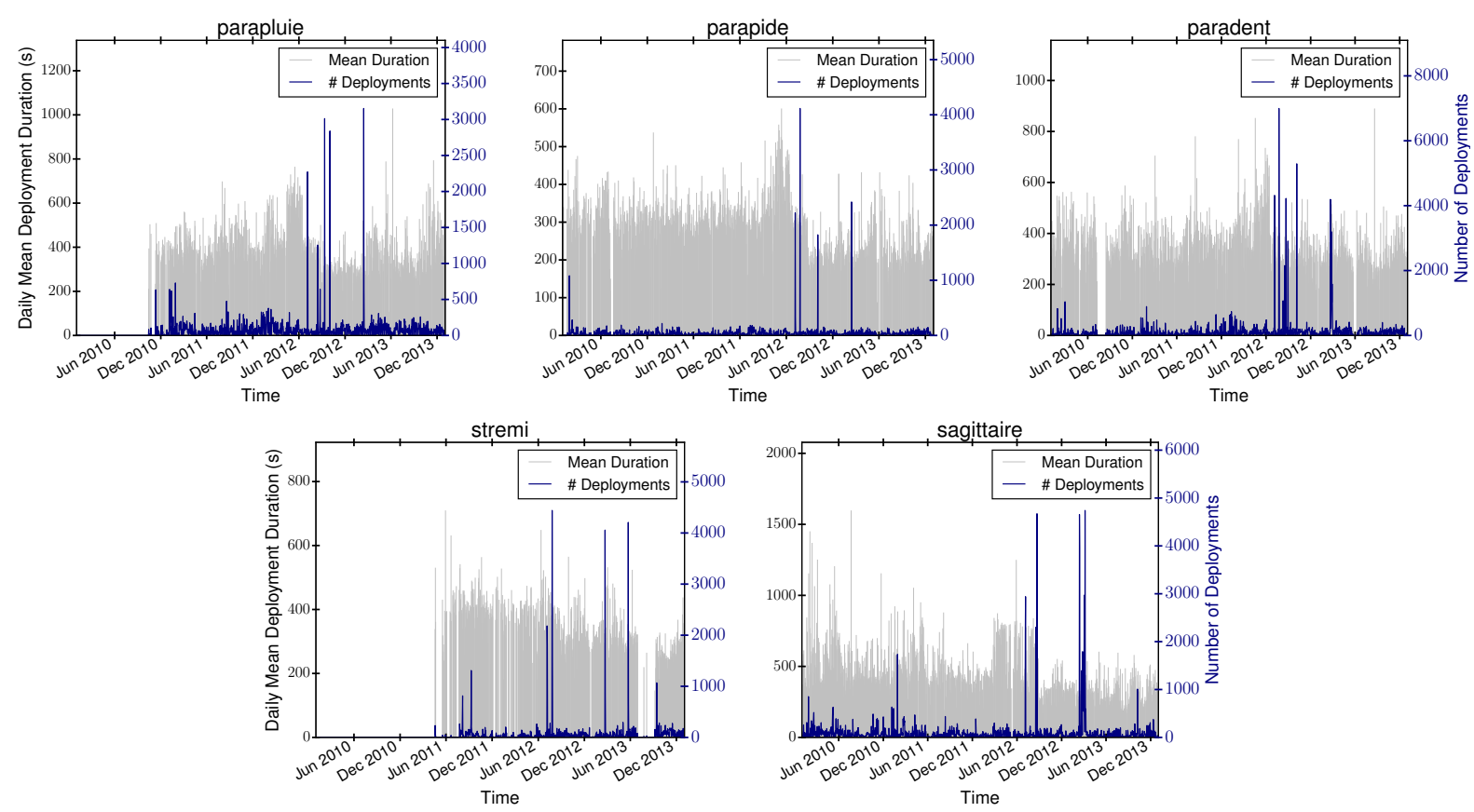

Fig. 4 Daily mean deployment duration and number of deployments over time for the various clusters.

The deployment time model is used in Section 5 to estimate how much time is required for initialising resources and configuring them to serve user requests.

\section{Resource Allocation Strategies}

This section discusses reservation strategies that a cloud provider can implement for reducing the energy consumed by computing resources, whereas their performance is investigated later in Section 5 .

\subsection{Power-Off Idle Resources}

This strategy checks resources periodically, and if a resource remains idle during a given time (i.e. idleness interval), it is powered off. As shown later, this strategy though simple and efficient from an energy consumption perspective, can lead to performance degradation if resources are continuously switched off or on.

\subsection{Reservation-Based Power-Off}

This approach is similar to powering-off idle resources, but when assessing a resource idleness, it also determines whether the resource is likely to remain unused over a time horizon. The strategy also initialises resources in advance to serve previously scheduled reservations. The average deployment time and a small safety margin are used to determine how long in advance resources must be deployed. Previous work evaluated lengths of idleness interval and the time horizon over which a server needs to remain unused to be considered a candidate to be switched off [20]. These intervals must be long enough so that frequent resource reinitialisation does not eclipse the potential energy savings achieved by powering idle resources off. Here idleness time and the horizon are 5 and 30 minutes respectively.

\subsection{Reservation With Minimum Capacity Estimation}

In addition to delaying when resources are made available to users, frequent server initialisation and shut down can be detrimental to energy efficiency. A typical server exhibits peaks of power consumption during boot and shut down phases [20], which we seek to avoid by using a technique proposed in our previous work [2] to configure a minimum resource pool, with a capacity below which decisions to switch resources off are ignored.

Resource utilisation is used for determining when the minimum capacity must be adjusted. Utilisation at time $t$, denoted by $v_{t}$, is the ratio between the number of resource hours used to handle requests and the number of hours resources were powered on (i.e., switched-off resources are not considered). The provider sets parameters $H$ and $L, 0 \leq L \leq H \leq 1$, indicating utilisation lower $(L)$ and upper $(H)$ thresholds according to which additional capacity is required or powered-on resources 
are not needed, respectively. In this work, $L=0.4$, $H=0.9$.

The minimum pool capacity should ideally be modified before utilisation reaches undesired levels, which requires a prediction on the future number of resources required. Here this estimation is based on the measurements performed over the past $i$ measurement intervals. Namely, after measuring $v_{t}$ at time $t$, weighted exponential smoothing is used to predict the utilisation for step $t+1$. If the past $v \leq i$ measurements (i.e., $v_{t-v}, v_{t-v+1}, \ldots, v_{t}$ ) and the forecast utilisation are below (above) the lower (upper) threshold $L(H)$, the minimum capacity must be adjusted. We employ $i=5$ and $v=10$. We use an exponential moving average of past numbers of required resources to compute the new minimum capacity.

\subsection{Exploiting Workload Periodicity}

Whereas strategies for aperiodic workloads are inherently more versatile as they can be applied to any workload, periodicity does provide valuable insights to adjust the number of resources that must be made available at a given time. We employ a technique derived from previous work [11] to explore workload periodicity and leverage information to determine the minimum number of resources required over the horizon.

The periodicity of a workload can be determined using historical data using techniques such as Fast Fourier Transform [11], autocorrelation and Discrete Fourier Transform [29]. The average utilisation from multiple periods can be used for estimating the minimum number of resources required during an interval.

The technique employed here creates variable-sized intervals instead of using fixed-length intervals. The technique simulates the scheduling of requests and determines the number of resources needed to handle the incoming request-rate. We measure the number of resources required to manage the load at fixed time steps, which provides us with a time series with the demand values at various time steps during a period. Then, we use K-means++ to classify the demand data points into groups to which they mostly resemble. We use $k=3$ considering three distinct load levels, namely low, medium and high.

Once measurement points have been clustered into demand-level groups, we walk through the demand time series and divide it into intervals in a manner that most measurements within an interval are in the same demand group. Whenever we find a sequence of measurements in a demand group followed by another sequence in a different group, we put the two sequences into distinct intervals. The minimum resource capacity required during each interval is the average of demand points obtained during the interval. More detailed information on the technique can be found in the literature [11].

This strategy hence uses a hybrid approach to configure a minimum pool of resources. It employs the load level intervals described above to set the minimum pool and at every time step $t$, adjusts the minimum capacity based on historical load and resource utilisation method described in Section 4.3.

\section{Performance Evaluation}

We evaluate the impact of reservations on the potential for energy savings and time to deliver servers to users.

\subsection{Experimental Setup}

A discrete-event simulator is used to model and simulate resource allocation and request scheduling ${ }^{2}$. As traces of cloud workloads with reservations are very difficult to come by, two sets of request traces were adapted to model cloud users' resource demands; one set extracted from Grid'5000's scheduler and another based on Google workload logs [22].

Despite using workloads from clusters, which may at first more related to private clouds, we believe that public clouds may present similar usage patterns. Systems with global presence when deployed on public clouds, use techniques such as redirection via DNS, content delivery optimisations, among others, to redirect clients to the nearest region/zone and hence minimise delays and improve response time. By doing so, each region may, in fact, be serving customers who are more geographically close and hence exhibit some of the diurnal patterns considered here.

\subsubsection{Grid'5000 Reservation Trace}

Request traces were collected from two Grid'5000 sites, Lyon and Reims, spanning six months, from Jan. 2014 to Jun. 2014. There are essentially two types of requests that users of Grid'5000 can make, namely reservations and best-effort; the latter is ignored in this work. Under normal operation, resource reservations are conditioned to available resources. For instance, a user willing to allocate resources for an experiment will often check a site's agenda, see what resources are available and will eventually make a reservation during a suitable time

\footnotetext{
2 https://github.com/assuncaomarcos/servsim
} 
frame. If the user cannot find enough resources, she will either relax her requirements - e.g. change the number of required resources, and reservation start or/and finish time - or choose another site with available capacity. The request traces, however, do not capture what the users' initial requirements were before they made their requests. For each site we consider the original trace, referred to as reservation, and another version termed as cloud where the original trace is modified as follows:

1. Requests whose original submission time is within working hours and start time lies outside these hours are considered on-demand requests starting at their original submission time.

2. Remaining requests are considered on-demand, both submitted and starting at their original start time.

3. The resource capacity of a site is modified to the maximum number of CPU cores required to honour all requests, plus a safety factor.

Change (1) adapts the behaviour of users who currently exploit resources during off-peak periods, whereas (2) alters the current practice of planning experiments in advance and reserving resources before they are taken by other users. Although the changes may seem extreme at first, they allow us to evaluate what we consider to be our worst case scenario where reservations are not possible. Moreover, as mentioned earlier, we believe that under the model adopted by existing clouds, where short-term reservations are not allowed, and prices of on-demand instances do not vary over time, users would have little incentives to exploit off-peak periods or plan their demand in advance. Change (3) reflects the industry practice of provisioning resources to handle peak demand including a safety margin.

\subsubsection{Google Workload Trace}

The original Google workload trace provides data over a month-long period in May 2011 from a 12k-machine set used by the Exploratory Testing Architecture [22]. The trace contains a log of job submissions, their schedule, and execution, where each job comprises one or multiple tasks that are executed on containers deployed on one or multiple machines. The original resource demands (e.g. memory, CPU, disk) are normalised by the configuration of the largest machine. To determine the number of physical machines $m_{j}$ that a job $j$ requires, we obtain the maximum set of simultaneous tasks in execution $T_{j}$ over the duration of job $j$. Then we compute $m_{j}=\min \left\{a, c * \sum_{t \in T_{j}} t / \operatorname{mac}_{t}^{m e m}\right\}$, where $\operatorname{mac}_{t}^{m e m}$ is the normalised capacity of the machine that executed task $t$; $c$ is a constant representing the available host's memory capacity allocated to containers, set to 0.85 ; and $a$ is a constant that specifies the maximum number of machines per request. The constant $a$ is set to 50 to prevent creating workloads that are extremely bursty and can hinder strategy comparison.

Certain jobs are very short and probably part of submission bursts for which we consider a user would make a single reservation. Hence jobs are grouped using a technique proposed for bag-of-tasks applications [12]. The continued submission grouping scheme is applied with $\Delta=180$ seconds as longer running jobs would more closely represent bare-metal deployment. From the original trace that contains jobs submitted by a total of 933 services, we crafted five different workloads, each comprising job submissions from 250 randomly selected services; these traces, depicted in Figure 5, are taken as the cloud workloads. As the original trace does not contain reservations, we create reservation workloads by randomly selecting requests that require reservations, where the reservation ratio varies as described later. Original job start time is used as the reservation start time and how long in advance the reservation is made is uniformly drawn from an interval of 0 to $24 \mathrm{~h}$.

\subsubsection{User Behaviour}

We believe that users of a cloud would plan their resource demands in advance and use reservations if enough incentives were provided. These incentives could materialise in the form of discount prices for resource allocation or information on how their behavioural changes affect resource allocation and maximise energy savings [20]. In this work, we do not focus on devising the proper incentives for users to adhere to reservations. The experiments consider that at least some users find enough incentives to change their allocation decisions and reserve resources in advance. A more detailed study on incentives is left for future work.

\subsubsection{Modelled Scenarios}

Two scenarios were modelled, where infrastructure capacity and resource requests are expressed in number of machines. The maximum number of machines available at each site is computed by simulating the request scheduling of their corresponding cloud workloads under a large number of machines, so that each request is treated as it arrives and no request is rejected. The maximum number of machines used during this evaluation is taken as the site capacity. Based on the deployment information from Kadeploy, we model the time in seconds required to boot powered-off machines requested by a reservation using a log-normal distribution 

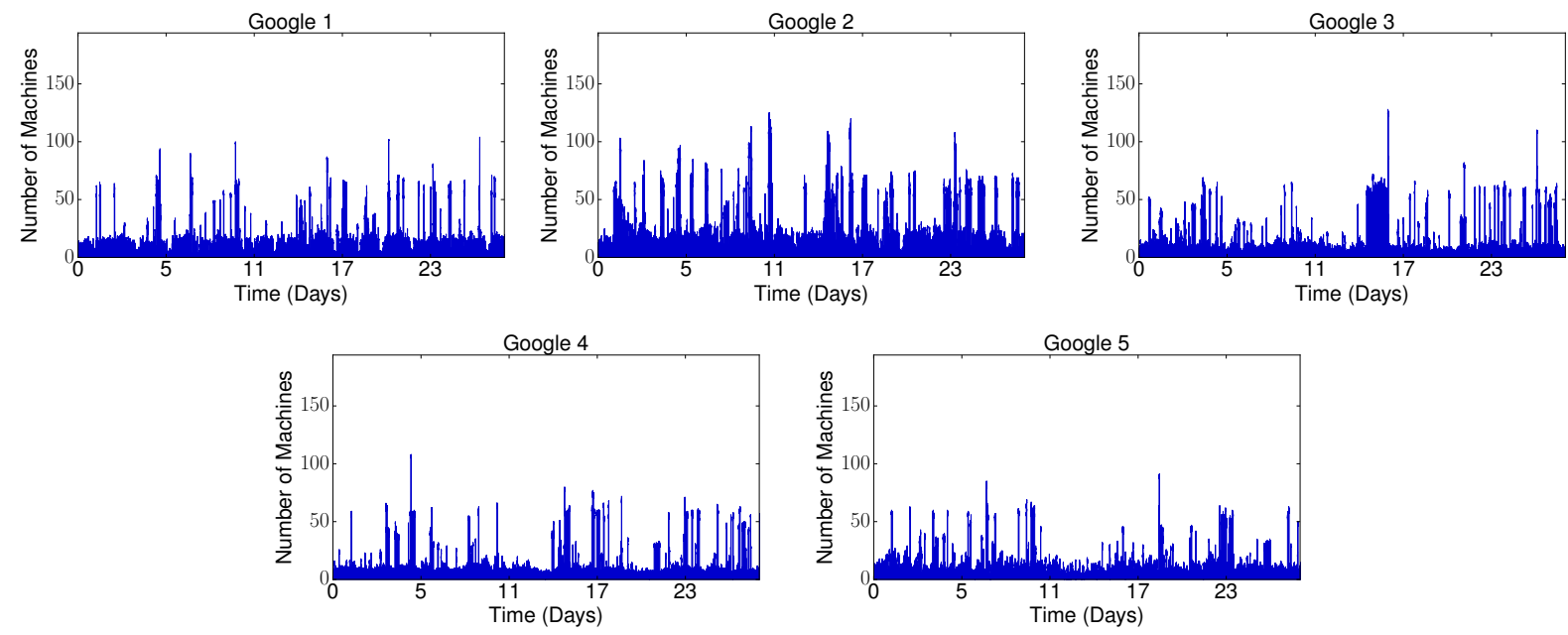

Fig. 5 Overview of Google cloud workloads.

whose scale is 6 and shape is 0.4 . We take 25 minutes as the time a machine must remain idle to be a candidate for switch off and 30 minutes as the future horizon to check whether it is committed to reservations. The evaluation of candidates for switch off is performed every 5 minutes.

To evaluate the potential energy savings resulting from including reservations, we considered the following schemes for resource provisioning:

- Cloud Always On: baseline scenario that uses the cloud workloads and maintains all servers constantly on. It is also used to determine the resource capacity to handle requests in an on-demand, cloudlike manner.

- Cloud Switch Off: does not consider reservations, employs the cloud workloads and the policy that switches servers off if they remain idle for a given interval.

- Reservation Switch Off: uses the reservation traces and the reservation policy that switches off servers that remain idle for an interval and that are not committed to requests over a time horizon. It also boots servers in advance to fulfil previously scheduled reservations.

- Reservation Minimum Pool: similar to the reservation with switch off but maintains a minimum resource pool available using the historic resource utilisation mechanism described in Section 4.3.

- Reservation Periodic: this strategy configures the minimum resource pool using the hybrid approach presented in Section 4.4. The first week of the workload traces is employed to compute the periodic load levels used to calculate the minimum resource pool.

\subsection{Performance Metrics}

Two metrics are considered, namely energy saving potential and request aggregate delay, which respectively measure how much of the total server idleness is used for server switch-off and how powering off resources affects the time to deliver requested servers to users.

\subsubsection{Energy Saving Potential}

The total server idleness si under the Cloud Always On scenario corresponds to the maximum time during which servers could potentially be switched off, and it is therefore considered the upper bound on potential energy savings. The si of a site is given by:

$s i=\int_{t_{0}}^{t_{\text {last }}} s_{\text {total }}-s_{\text {used }} d t$

where $t_{0}$ is the start of the evaluation, $t_{\text {last }}$ is when the last request is submitted, $s_{\text {total }}$ is the total number of servers available at any time, and $s_{\text {used }}$ is the number of machines in use at time $t$. The potential for energy saving is the percentage of si during which servers are switched off.

\subsubsection{Request Aggregate Delay}

This metric quantifies the impact that switching servers off has on the QoS users perceive; it measures the time users have to wait to have their requests serviced. The aggregate delay ad of requests whose Quality of Service (QoS) has been impacted $\left(R_{\text {delay }}\right)$ is given by:

$a d=\sum_{r \in R_{\text {delay }}} r_{\text {deploy_end }}-r_{\text {start_time }}$ 
where $r_{\text {deploy_end }}$ is when the last server became ready to use, and $r_{\text {start_time }}$ is the time when the request was supposed to start; that is, when the user expected the servers to be available. The distributions of deployment time obtained while inspecting Kadeploy traces give a conservative estimate to evaluate $a d$. Certain public cloud providers publicise provisioning times of bare-metal resources much higher than those found in Grid'5000.

\subsection{Evaluation Results}

Figures 6 and Figure 7 summarise the results on potential energy savings and request aggregate delay. Here $40 \%$ of requests of each Google workload are randomly selected to require reservations. As the Cloud Switch Off scheduling strategy switches servers off almost immediately once it determines that they have remained idle, it is able to achieve higher energy saving potential. This simple policy, however, does not consider the cost of powering off/on resources and hence presents the largest request aggregate delay. The Reservation Switch Off scenario, on the other hand, exploits less idle time, but as shown in Figure 7 leads to smaller QoS degradation. In addition, reservation provides some advantages with respect to peak capacity planning. For instance, when handling requests in a cloud manner, Lyon and Reims Grid'5000 sites require peak capacities of respectively 194 and 136 machines; when accepting reservations, the peak capacities are respectively 89 and 78 servers, which is over $45 \%$ less servers than the Cloud scenario.

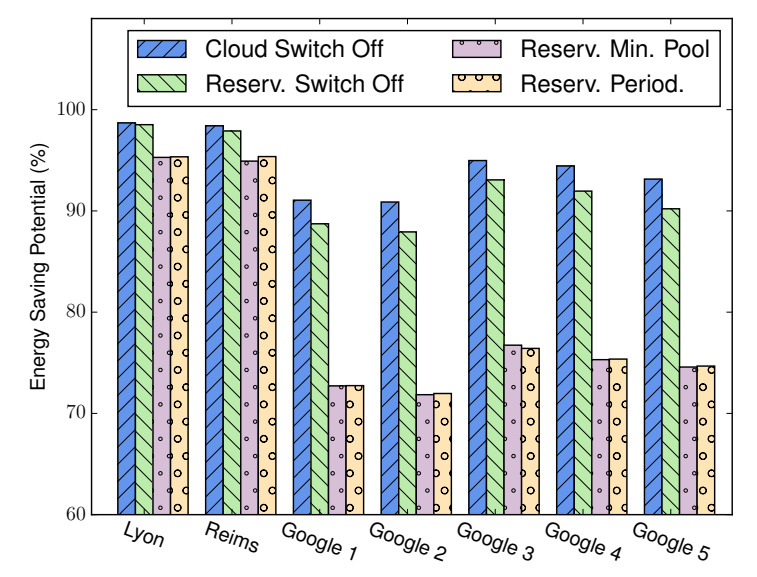

Fig. 6 Energy saving potential for the various scenarios.

As shown in Figure 6, reservation with a minimum resource pool presents smaller energy savings compared to the simple reservation strategy, but it reduces the request aggregate delay. In the case of Google workloads, although reservation reduces the aggregate request delay as shown in Figure 7, this reduction results in smaller energy saving potential. Further investigation of this issue revealed that the exponential smoothing applied to forecast required server capacity leads to a minimum number of resources being kept on during longer periods than under other strategies. Although at first one may assume that other strategies can always better exploit the bursty behaviour of the Google workloads, it is important to note that we use conservative bare-metal deployment times. We believe that when considering the times reported by the industry - where servers take several hours to be provisioned or recycled - the smoother behaviour of reservation with minimum pool is preferable. When compared to Reservation Minimum Pool, the Reservation Periodic strategy achieves very minor gains in potential energy savings under certain workloads, but it worsens the aggregate request delay. The reason for the greater request delay is that under certain cases the minimum resource pool is set to a larger number than what is in fact used, thus resulting in lower resource utilisation. This lower utilisation affects the size of the minimum pool over subsequent periods when the periodic approach demands a low number of resources and sudden request bursts arise.

In order to evaluate the impact of using reservation on the request aggregate delay, we performed another experiment with the Google workloads by varying the reservation ratio from 0.1 to 0.9 (i.e. from $10 \%$ to $90 \%$ of requests are selected to require reservations). Figure 8 shows that in general the aggregate delay is inversely proportional to the reservation ratio. It is important to highlight that the impact of reservations on the aggregate request delay could have been higher if grouped jobs from the original trace had not been grouped to build what we believe is a more realistic scenario of bare-metal deployment.

\section{Related Work}

The benefits and drawbacks of resource reservations have been extensively studied for various systems, such as clusters of computers $[13,15,17,23,25]$, meta-schedulers [26], computational grids $[7,8,10]$, virtual clusters and virtual infrastructure [6]; and have been applied under multiple scenarios including co-allocation of resources [19], and improving performance predictability of certain applications [32].

Smith et al. [25] evaluated the impact of including support for advance reservations on scheduling systems. They concluded that the mean wait time of submitted 


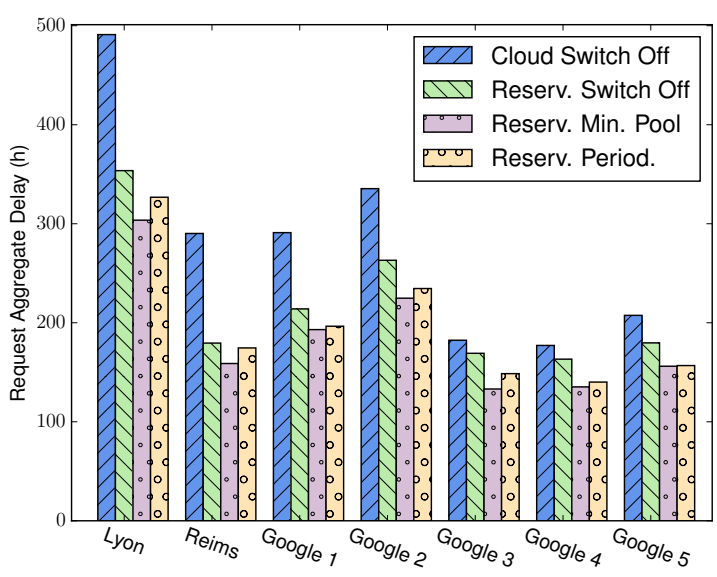

Fig. 7 Request aggregate delay in resource/hour.

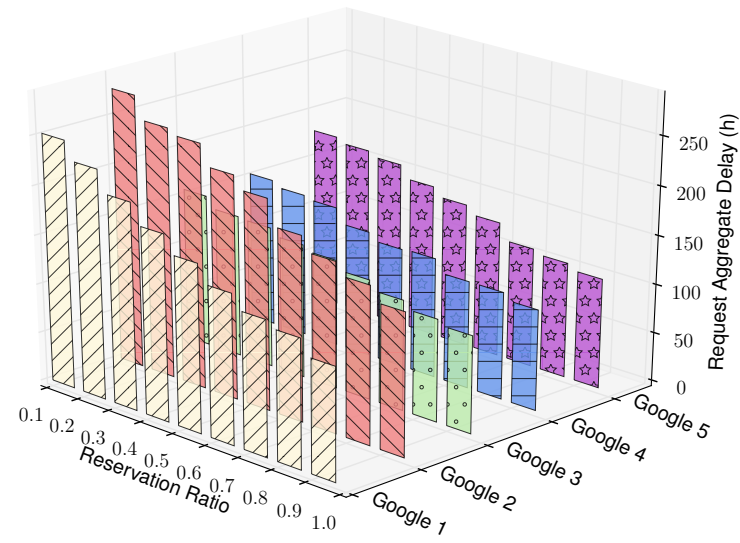

Fig. 8 Request delay in resource/hour for Google workloads.

applications might increase when supporting reservations and that the increase depends on how reservations are supported (i.e. whether queued requests have priority or not). They also identify that reservations may lead to fragmentation of system resources.

Margo et al. [17] also confirm that wait times can increase, but highlight the needs for reservations when coallocating resources from multiple sites for applications such as workflows [32]. They also describe techniques to mitigate the impact of reservations on traditional management. The need for reservations as a means to enable jobs that require resource co-allocation is also highlighted by Snell et al. [26] who describe algorithms for scheduling meta-jobs. A brokering service also capable of performing meta-scheduling and supporting reservations is described by Elmroth and Tordsson [7].

Lawson and Smirni [15] aim at reducing fragmentation by providing a non-FCFS policy that schedules jobs using multiple queues. Farooq et al. [8] attempt to reduce the performance cost of reservations by adding laxity to the reservation window and hence giving more flexibility to the scheduling system. Flexible or lax reservations is a concept also explored by Netto et al. to support resource co-allocation [19].

Previous work also provided techniques for placing reservations into a schedule. Approaches such as those provided by Röblitz and Rzadca [23] attempt to decide on the placement of a reservation by executing what-if schedules in case the reservation is accepted. The whatif scenarios often simulate backfilling scheduling schedules trying to (re)accommodate the already accepted jobs.

Most previous work, however, has either ignored the time to provision reserved resources or employed provisioning models that not always reflect reality. Shirako [13] is a system that considers the setup and teardown phases of resource provisioning. Reservation users often bear the costs of resource setup leaving teardown costs to successors (i.e. the next users to use the resources). The work, however, does not attempt to model the time required to deploy bare-metal resources.

Amazon Web Services (AWS) ${ }^{3}$ offers cloud services that suit several of today's use cases and provides the richest set of reservation options for VM instances. AWS offers four models for allocating VM instances, namely on-demand, reserved instances, spot instances and dedicated; the latter are allocated within a Virtual Private Cloud (VPC). Under all models AWS allows users to request HPC instances, optimised for processing, memory use, I/O, and instances with Graphical Processing Units (GPUs). Reserved instances can be requested at a discount price under the establishment of long-term contracts. Dedicated instances, provided at a premium, are those that most closely resemble bare-metal provisioning, as two instances requested by a given service do not share a physical host.

Wang et al. [31] have exploited the use of reservations in the cloud by considering a cloud brokerage service that reserves a large set of VM instances from cloud providers and the serves user requests at discount price. While considering a cloud provider's revenue maximisation, Toosi et al. [28] present a stochastic dynamic programming technique for determining the maximum number of reservations that the provider can accept. The present work evaluates the energy savings that could be achieved if short-term reservations were accepted, and how the time required to configure the resources affect how long it takes for the resources to become available to reservation users.

OpenNebula [18] and Haizea [27] support multiple types of reservations (e.g. immediate, advanced and

3 http://aws.amazon.com/ec2/ 
best-effort) and consider the time required to prepare and configure the resources used by VMs (e.g. time to transfer VM images). Climate, a reservation system conceived during the FSN XLCloud ${ }^{4}$ project, and later renamed Blazar when incorporated into OpenStack ${ }^{5}$ enables reserving and deploying bare-metal resources whilst taking into account their energy efficiency [3].

\section{Conclusion and Future Work}

This article discussed the impact of reservation support in the provision of bare-metal resources in Clouds. The work analysed historical information on the deployment of bare-metal resources and evaluated strategies for switching off servers in a cloud data centre. Results show the impact of adding resource reservations on the number of machines required to handle peak load and the time used to deploy environments such as operating system and software stack required by customers. Under the evaluated environments and workloads, reservations can reduce the time to deliver resources to users when compared to allocation strategies that naively switch off idle servers. Moreover, when using reservations, the maximum number of machines required to handle peak load at the examined Grid'5000 sites was over $45 \%$ smaller compared to handling requests in an on-demand, cloud-like fashion.

In future work, we would like to gain more insights on user behaviour on how they would exploit reservation of bare-metal resource in the cloud. We would like to obtain request traces that can tell us more about users' habits and the patterns of resource requests. We also intend to conduct user surveys to understand how users would utilise short-term reservations.

\section{Acknowledgements}

This research is partially supported by the CHIST-ERA STAR project. Some experiments presented in this paper were carried out using the Grid'5000 experimental testbed, being developed under the Inria ALADDIN development action with support from CNRS, RENATER and several Universities as well as other funding bodies (see https://www.grid5000.fr).

\section{References}

1. Armbrust, M., Fox, A., Griffith, R., Joseph, A.D., Katz, R.H., Konwinski, A., Lee, G., Patterson, D.A., Rabkin,

\footnotetext{
${ }^{4}$ http://xlcloud.org

5 https://wiki.openstack.org/wiki/Blazar

A., Stoica, I., Zaharia, M.: Above the clouds: A Berkeley
}

view of Cloud computing. Tech. Report UCB/EECS2009-28, Electrical Engineering and Computer Sciences, University of California at Berkeley, Berkeley, USA (2009)

2. Assuncao, M.D., Cardonha, C.H., Netto, M.A., Cunha, R.L.F.: Impact of user patience on auto-scaling resource capacity for cloud services. Future Generation Computer Systems 55, 41-50 (2016). DOI http://dx.doi.org/10. 1016/j.future.2015.09.001

3. Assuncao, M.D., Lefevre, L., Rossigneux, F.: On the impact of advance reservations for energy-aware provisioning of bare-metal cloud resources. In: 12th International Conference on Network and Service Management (CNSM 2016) (2016)

4. Bolze, R., Cappello, F., Caron, E., Daydé, M., Desprez, F., Jeannot, E., Jégou, Y., Lantéri, S., Leduc, J., Melab, N., Mornet, G., Namyst, R., Primet, P., Quetier, B., Richard, O., Talbi, E.G., Iréa, T.: Grid'5000: a large scale and highly reconfigurable experimental Grid testbed. Int. J. of High Perf. Comp. Applications 20(4), 481-494 (2006)

5. Capit, N., Costa, G.D., Georgiou, Y., Huard, G., Martin, C., Mounié, G., Neyron, P., Richard, O.: A batch scheduler with high level components. In: CCGrid'05, vol. 2, pp. 776-783. Washington, USA (2005)

6. Chase, J.S., Irwin, D.E., Grit, L.E., Moore, J.D., Sprenkle, S.E.: Dynamic virtual clusters in a Grid site manager. In: HPDC 2003, p. 90. Washington, USA (2003)

7. Elmroth, E., Tordsson, J.: A standards-based Grid resource brokering service supporting advance reservations, coallocation, and cross-Grid interoperability. $\mathrm{CCPE}$ 21(18), 2298-2335 (2009)

8. Farooq, U., Majumdar, S., Parsons, E.W.: Impact of laxity on scheduling with advance reservations in Grids. In: MASCOTS 2005, pp. 319-322 (2005)

9. Feitelson, D.G., Tsafrir, D., Krakov, D.: Experience with the parallel workloads archive. JPDC To appear

10. Foster, I., Kesselman, C., Lee, C., Lindell, B., Nahrstedt, K., Roy, A.: A distributed resource management architecture that supports advance reservations and coallocation. In: IWQoS'99, pp. 27-36. London, UK (1999)

11. Gandhi, A., Chen, Y., Gmach, D., Arlitt, M., Marwah, M.: Hybrid resource provisioning for minimizing data center SLA violations and power consumption. Sustainable Comp.: Informatics and Systems 2(2), 91-104 (2012)

12. Iosup, A., Jan, M., Sonmez, O., Epema, D.: The characteristics and performance of groups of jobs in grids. In: A.M. Kermarrec, L. Bouge, T. Priol (eds.) Euro-Par 2007 Parallel Processing, LNCS, vol. 4641, pp. 382-393. Springer (2007)

13. Irwin, D., Chase, J., Grit, L., Yumerefendi, A., Becker, D., Yocum, K.G.: Sharing networked resources with brokered leases. In: USENIX Annual Technical Conf., pp. 199-212. Berkeley, USA (2006)

14. Jeanvoine, E., Sarzyniec, L., Nussbaum, L.: Kadeploy3: Efficient and Scalable Operating System Provisioning. USENIX ;login: 38(1), 38-44 (2013)

15. Lawson, B.G., Smirni, E.: Multiple-queue backfilling scheduling with priorities and reservations for parallel systems. In: JSSPP 2002, LNCS, pp. 72-87. Springer, London, UK (2002)

16. Marathe, A., Harris, R., Lowenthal, D.K., de Supinski, B.R., Rountree, B., Schulz, M., Yuan, X.: A comparative study of high-performance computing on the cloud. In: HPDC 2013, pp. 239-250. ACM, New York, USA (2013) 
17. Margo, M.W., Yoshimoto, K., Kovatch, P., Andrews, P.: Impact of reservations on production job scheduling. In: JSSPP 2007, LNCS, vol. 4942, pp. 116-131. Springer, Berlin/Heidelberg, Germany (2007)

18. Milojicic, D., Llorente, I.M., Montero, R.S.: OpenNebula: A cloud management tool. IEEE Internet Comp. 15(2), 11-14 (2011)

19. Netto, M.A.S., Bubendorfer, K., Buyya, R.: SLA-Based advance reservations with flexible and adaptive time QoS parameters. In: 5th Int. Conf. on Service-Oriented Comp. (ICSOC 2007), pp. 119-131. Springer, Berlin, Heidelberg, Germany (2007)

20. Orgerie, A.C., Lefèvre, L., Gelas, J.P.: Save watts in your Grid: Green strategies for energy-aware framework in large scale distributed systems. In: ICPADS'08, pp. 171-178. Melbourne, Australia (2008)

21. Ostermann, S., Iosup, A., Yigitbasi, N., Prodan, R., Fahringer, T., Epema, D.: A performance analysis of EC2 cloud computing services for scientific computing. In: Cloud Comp., LNCS, vol. 34, pp. 115-131. Springer (2010)

22. Reiss, C., Wilkes, J., Hellerstein, J.L.: Google clusterusage traces: Format + schema. Tech. report, Google Inc., Mountain View, USA (2011)

23. Röblitz, T., Rzadca, K.: On the placement of reservations into job schedules. In: Euro-Par 2006 Parallel Processing, LNCS, vol. 4128, pp. 198-210. Springer (2006)

24. Scott, D.W.: On optimal and data-based histograms. Biometrika 66(3), 605-610 (1979)

25. Smith, W., Foster, I., Taylor, V.: Scheduling with advanced reservations. In: 14th Int. Parallel and Dist. Processing Symp. (IPDPS 2000), pp. 127-132. Cancun, Mexico (2000)

26. Snell, Q., Clement, M., Jackson, D., Gregory, C.: The performance impact of advance reservation meta-scheduling.
In: JSSPP 2000, LNCS, vol. 1911, pp. 137-153. Springer (2000)

27. Sotomayor, B., Keahey, K., Foster, I.: Combining batch execution and leasing using virtual machines. In: HPDC 2008, pp. 87-96. New York, USA (2008)

28. Toosi, A.N., Vanmechelen, K., Ramamohanarao, K., Buyya, R.: Revenue maximization with optimal capacity control in infrastructure as a service cloud markets. IEEE Transactions on Cloud Computing 3(3), 261-274 (2015). DOI 10.1109/TCC.2014.2382119

29. Verma, A., Dasgupta, G., Nayak, T.K., De, P., Kothari, R.: Server workload analysis for power minimization using consolidation. In: USENIX Annual Technical Conference, p. 28. USENIX Association (2009)

30. Wang, G., Ng, T.S.E.: The impact of virtualization on network performance of Amazon EC2 data center. In: 29th Conf. on Information Communications (INFOCOM'10), pp. 1163-1171. IEEE Press, Piscataway, USA (2010)

31. Wang, W., Niu, D., Liang, B., Li, B.: Dynamic cloud instance acquisition via iaas cloud brokerage. IEEE Transactions on Parallel and Distributed Systems 26(6), 1580 1593 (2015). DOI 10.1109/TPDS.2014.2326409

32. Wieczorek, M., Siddiqui, M., Villazon, A., Prodan, R., Fahringer, T.: Applying advance reservation to increase predictability of workflow execution on the Grid. In: 2nd IEEE Int. Conf. on e-Science and Grid Comp. (E-Science 2006), p. 82. Washington, USA (2006)

33. Younge, A.J., Henschel, R., Brown, J.T., von Laszewski, G., Qiu, J., Fox, G.C.: Analysis of virtualization technologies for high performance computing environments. In: IEEE Int. Conf. Cloud Comp. (CLOUD 2011), pp. 9-16 (2011) 